Fissile and Non-Fissile Material Detection using Nuclear Acoustic Resonance Signatures

J. Herberg, R. Maxwell, B. R. Tittmann, P. M. Lenahan, S. Yerkes, S. Jayaraman

October 12, 2005 
This document was prepared as an account of work sponsored by an agency of the United States Government. Neither the United States Government nor the University of California nor any of their employees, makes any warranty, express or implied, or assumes any legal liability or responsibility for the accuracy, completeness, or usefulness of any information, apparatus, product, or process disclosed, or represents that its use would not infringe privately owned rights. Reference herein to any specific commercial product, process, or service by trade name, trademark, manufacturer, or otherwise, does not necessarily constitute or imply its endorsement, recommendation, or favoring by the United States Government or the University of California. The views and opinions of authors expressed herein do not necessarily state or reflect those of the United States Government or the University of California, and shall not be used for advertising or product endorsement purposes.

This work was performed under the auspices of the U.S. Department of Energy by University of California, Lawrence Livermore National Laboratory under Contract W-7405-Eng-48. 


\title{
Fissile and Non-Fissile Material Detection using Nuclear Acoustic Resonance Signatures
}

\author{
Julie Herberg, Robert Maxwell \\ Center for National Security Applications of Magnetic Resonance \\ Chemistry and Materials Science and Engineering Directorates \\ Lawrence Livermore National Laboratory \\ Livermore, CA 94550
}

Prof. Bernhard R. Tittmann, Prof. Pat M. Lenahan, Shane Yerkes, and Subash B. Jayaraman

Department of Engineering Science and Mechanics

The Pennsylvania State University

University Park, PA

October 3, 2005 


\section{Executive Summary}

This report reviews progress made on NA22 project LL251DP to develop a novel technique, Nuclear Acoustic Resonance (NAR), for remote, non-destructive, nonradiation-based detection of materials of interest to Nonproliferation Programs, including ${ }^{235} \mathrm{U}$ and ${ }^{239} \mathrm{Pu}$. We have met all milestones and deliverables for FY05, as shown in Table 1. In short, we have developed a magnetic shield chamber and magnetic field, develop a digital lock-in amplifier computer to integrate both the ultrasound radiation with the detector, developed strain measurements, and begin to perform initial measurements to obtain a NAR signal from aluminum at room temperature and near the earth's magnetic field. The results obtained in FY05 further support the feasibility of successful demonstration of an NAR experiment for remote, non-destructive, non-radiation-based detection of materials of interest to Nonproliferation Programs.

Table 1. FY05 Milestones and Deliverables for LL251DP

\begin{tabular}{|l|l|l|l|}
\hline Due date & M/D & Description & Status \\
\hline $04 / 15 / 2005$ & $\mathrm{M} / \mathrm{D}$ & Development of SQUID detectors & Completed \\
\hline $09 / 30 / 2005$ & $\mathrm{M} / \mathrm{D}$ & $\begin{array}{l}\text { Development of SQUID detectors to investigate how } \\
\text { much ultrasonic energy is needed to drive the nuclei and } \\
\text { electrons in a particular sample }\end{array}$ & Competed \\
\hline $09 / 30 / 2005$ & $\mathrm{M}$ & $\begin{array}{l}\text { Begin to develop LabView to integrate both the } \\
\text { ultrasound radiation with the detectors }\end{array}$ & Completed \\
\hline $05 / 15 / 2006$ & $\mathrm{M} / \mathrm{D}$ & $\begin{array}{l}\text { Development of a magnetic shield chamber and magnetic } \\
\text { field }\end{array}$ & Completed \\
\hline $05 / 15 / 2006$ & $\mathrm{M} / \mathrm{D}$ & $\begin{array}{l}\text { Begin to develop techniques to performing NAR with the } \\
\text { Potassium Magnetometer }\end{array}$ & Completed \\
\hline $05 / 15 / 2006$ & $\mathrm{M}$ & Development of a digital lock-in amplifier computer & Completed \\
\hline $09 / 15 / 2005$ & $\mathrm{M} / \mathrm{D}$ & $\begin{array}{l}\text { Development of strain measurements } \\
\text { Completed }\end{array}$ \\
\hline $09 / 15 / 2005$ & $\mathrm{M} / \mathrm{D}$ & $\begin{array}{l}\text { Development of theoretical techniques to } \\
\text { understand the ultrasound affects }\end{array}$ & Completed \\
\hline $05 / 15 / 2006$ & $\mathrm{M} / \mathrm{D}$ & Double resonance experiments & On schedule \\
\hline
\end{tabular}

\section{Introduction:}

We are developing a novel technique, Nuclear Acoustic Resonance (NAR), for remote, non-destructive, non-radiation-based detection of materials of interest to Nonproliferation Programs, including ${ }^{235} \mathrm{U}$ and ${ }^{239} \mathrm{Pu}$. This new technique uses the unique NAR signatures generated by acoustic excitation of phonon modes via ultrasound. The acoustic modulation of internal interactions involving the magnetic and electric multipole moments of the nucleus is potentially observable by nuclear or electron spin transitions. Our approach takes advantage of collaboration with The Pennsylvania State University (PSU), which has expertise in ultrasound and electron spin resonance, along with LLNL's expertise in Nuclear Magnetic Resonance (NMR). The current collaboration between PSU and LLNL will focus on producing a definitive initial estimate of the advantages and limitations of this approach for materials containing ${ }^{235} \mathrm{U}$ 
and ${ }^{239} \mathrm{Pu}$. If successful, the project will be transferred to LLNL, where NAR techniques will be performed on actual samples of special nuclear materials. We are in the final stages of estimating the advantage and limitations of this approach and plan to conclude this phase of the project in FY06. Since this technique does not rely on nuclear radiation detection, the eventual device objective would be to detect and identify fissile materials even if steps had been taken by smugglers to completely shield the materials as a radiation source. A successful device could have enormous potential for the detection and identification of concealed fissile materials, a key and still unresolved issue facing nonproliferation and counterterrorism.

\section{Progress:}

In this report, we are addressing major achievements in developing an an NAR experiment for remote, non-destructive, non-radiation-based detection of materials of interest to Nonproliferation Programs, which include:

- developing a magnetic shield chamber and magnetic field to cut down on background noise and slightly increase the earth's magnetic field (Appendix A)

- developing a digital lock-in amplifier computer to integrate both the ultrasound radiation with the detector (Appendix B)

- performed strain measurements to determine the amount of acoustic wave we need to drive the electronic and nuclear interactions. (Appendix $C$ )

- begun to perform initial measurements to obtain an NAR signal for aluminum at room temperature and near the earth's magnetic field. (Appendix D)

\section{Outlook / Conclusions:}

In FY05, we constructed a magnetic shield chamber and magnetic field to cut down on background noise and slightly increase the earth's magnetic field and got the potassium magnetometer up and running. In addition, we have performed strain measurements to determine the amount of acoustic wave we need to drive the electronic and nuclear interactions. This has all led to developing the initial measurements to obtain an NAR signal for aluminum at room temperature and near the earth's magnetic field. All data to date continues to suggest that we can be successful. 


\section{Appendix A: Developing a magnetic shield chamber and magnetic field to cut down on background noise and slightly increase the earth's magnetic field}

We have determined that both the Overhauser and potassium magnetometer require a very stable and homogeneous field in order to minimize the background noise. We have researched magnetically shielded chambers and carried out fairly extensive calculations on the (very highly uniform) magnetic fields which can be generated within a long cylindrical high permeability chamber with a solenoid. With the help of Ammuneal Corp., we have developed and obtained a three-walled magnetically shielded chamber, which is shown in Figure 1. Students at PSU in Professor Lenahan's group have designed and constructed a simple, relatively inexpensive temperature compensated Hall Effect probes to monitor the small ambient AC and DC magnetic fields outside the magnetically shielded chamber. Students are also working on a magnetic field controller for the shielded chamber/solenoid/magnetometer system. These students have also built a large solenoid, which is shown in Figure 2, to produce an extremely uniform static magnetic field with a field strength of 1.78 Gauss. Along with this large solenoid, these students have designed and built a circuit to provide a steady current supply for the uniform magnetic field.

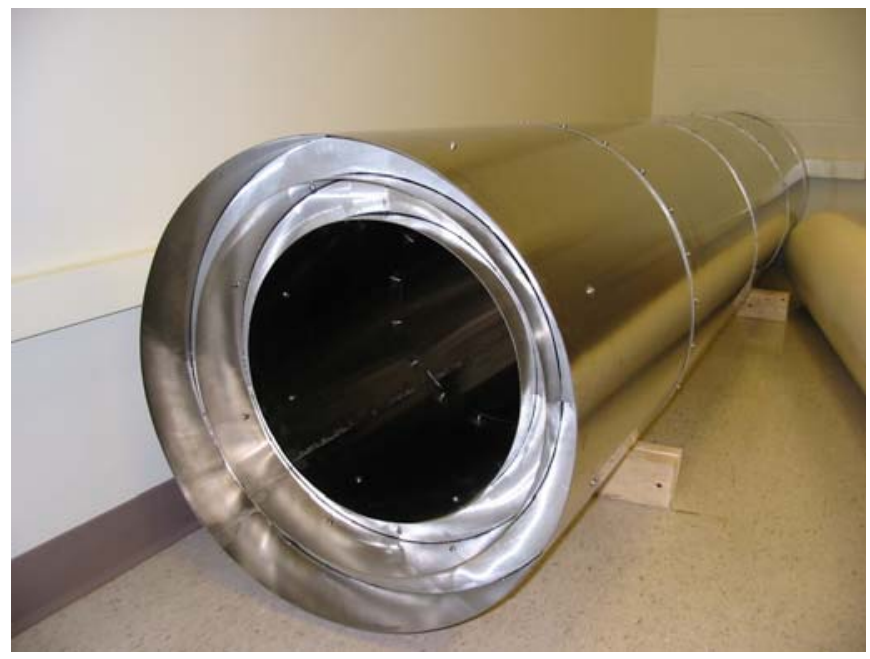

Figure 1: Triple magnetically shielded chamber.

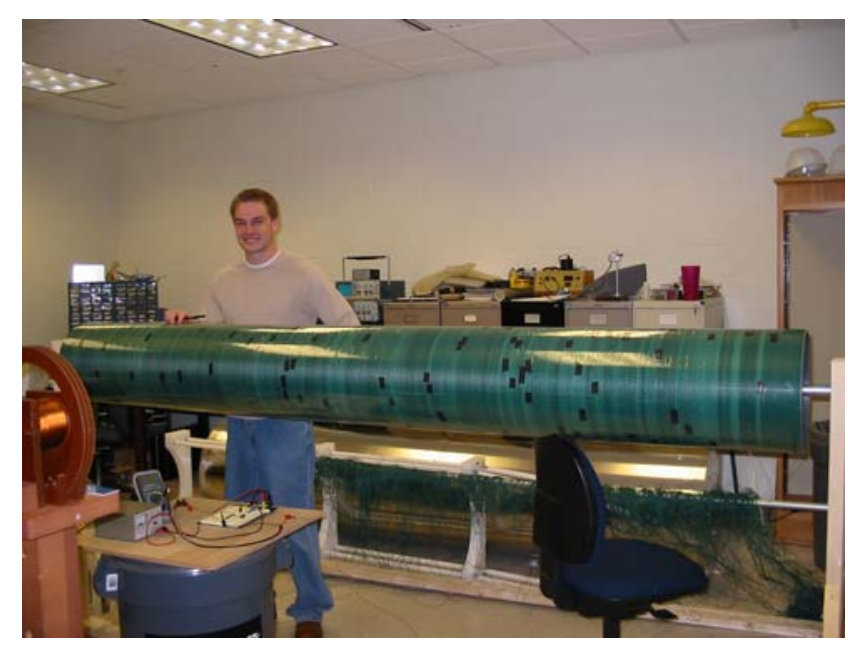

Figure 2: Extremely uniform static magnetic field with a magnetic field of 1.78 Gauss. 


\section{Appendix B: Developing a digital lock-in amplifier computer to integrate both the ultrasound radiation with the detector}

A digital lock-in amplifier has been implemented in software using the Lab View environment. This software controlled lock-in amplifier has many advantages over the traditional lock-in amplifier equipment for our acoustic magnetic resonance applications. First of all, this system is portable. Only a computer and a Data Acquisition System (DAQ) are required for operation. Secondly, a Graphical User Interface (GUI) was created for easy use. With the incorporation of a GUI, the user can adjust the modulation frequency, the modulation amplitude, the modulation phase, the bandpass filter width, and the time constant of the low pass filter. Also, the signal amplitude and xy plot are implemented for visual feedback. The GUI can be seen in Figure 3. A third advantage of this software controlled lock-in amplifier is that settings can be saved to the computer for multiple users. This is useful for the case where multiple experiments are performed using the same acquisition system.

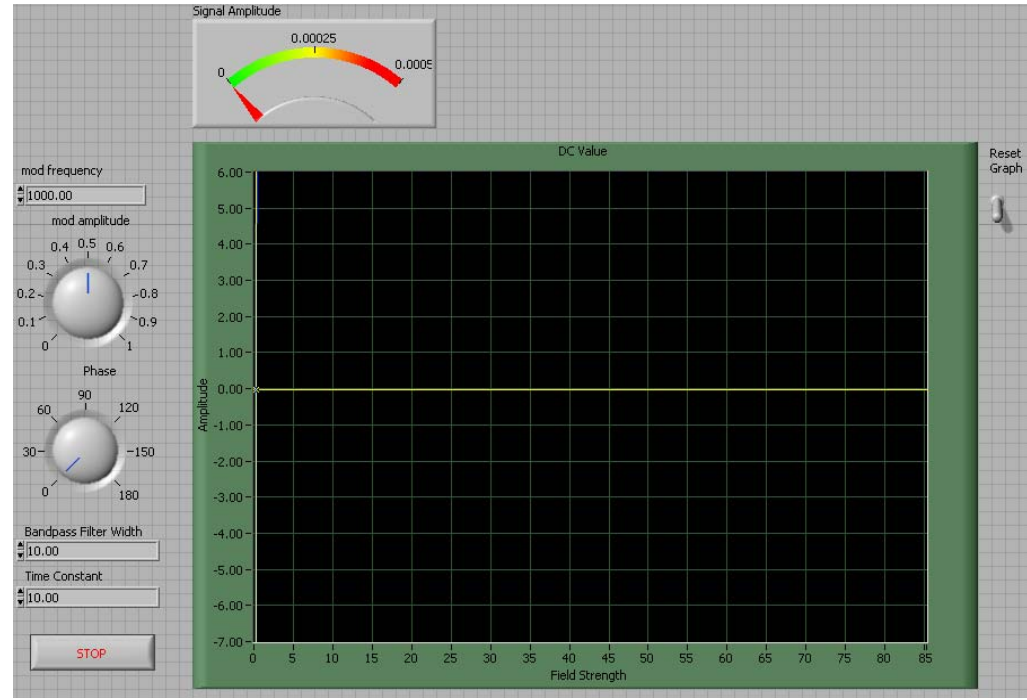

Figure 3: Graphical User Interface of the Digital Lock-in Amplifier

The basic block diagram that represents the function of the digital lock-in can be seen in Figure 4. The main components of the lock-in amplifier are the voltage controlled oscillator, AC amplifier, a multiplier, a low pass filter, and a DC amplifier. All of these functions are simulated and controlled digitally through Lab View. 


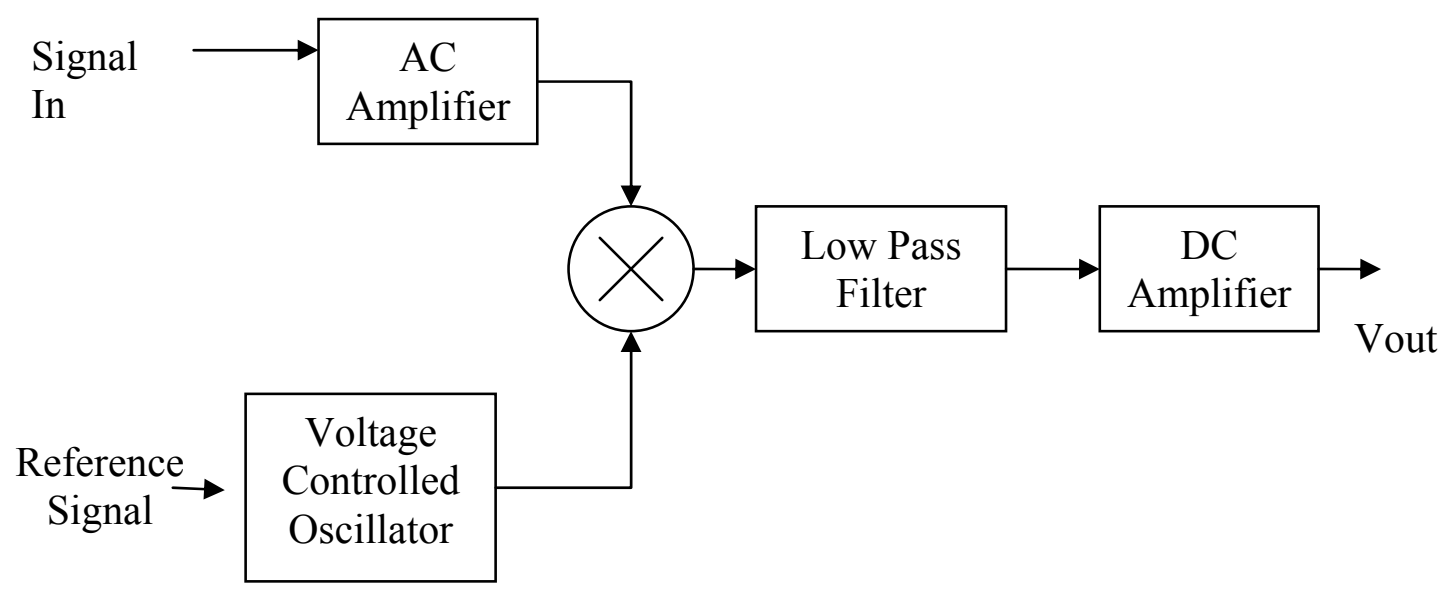

Figure 4: This figure represents the block diagram of a traditional lock-in.

In summary, a digital lock-in amplifier is more advantageous than the traditional lock-in amplifiers. This lock-in amplifier is extremely useful and easy to use; it can provide data acquisition at essentially arbitrary modulation frequencies and time constants, making it particularly useful for our acoustic magnetic resonance studies. 


\section{Appendix C: Performed strain measurements to determine the amount of acoustic wave we need to drive the electronic and nuclear interactions}

Experiments were conducted at Penn State by Prof. Tittmann and Subash Jayaraman, to measure the amount of strain being imparted to a polycrystalline Aluminum cylinder (10 cm long, $3.2 \mathrm{~cm}$ in diameter), using piezoelectric transducers. A Polytec ${ }^{\circledR}$ OFV-505 Laser Doppler Vibrometer (LDV) was used to observe the displacements at the cylinder face. Information regarding the vibrometer principles and operation could be found at www.polytec.com.

From ENDOR tables, the nuclear and electron spin resonance frequencies for Aluminum, in earth's magnetic field $(0.545 \mathrm{G})$, were calculated to be $599.57 \mathrm{~Hz}$ and $1.511 \mathrm{MHz}$ respectively.
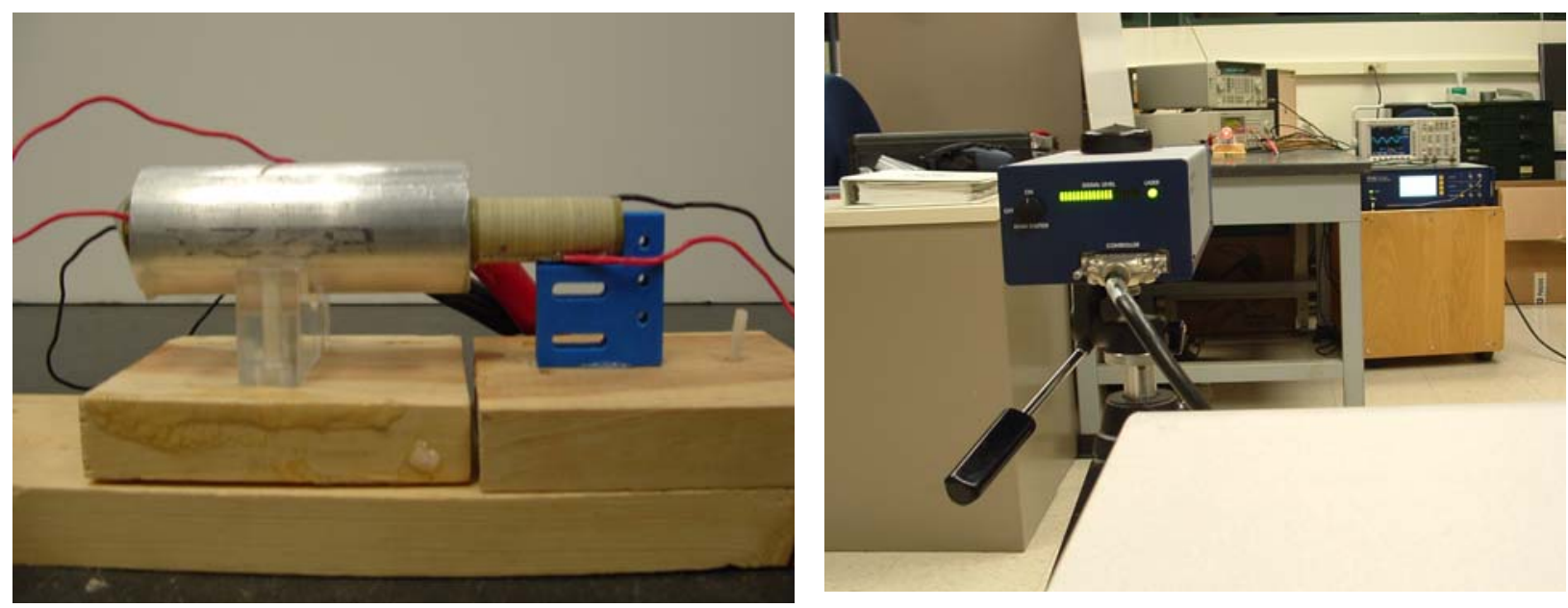

Figure 5. (a) Close-up of the set-up (b) Overall view of the experimental setup

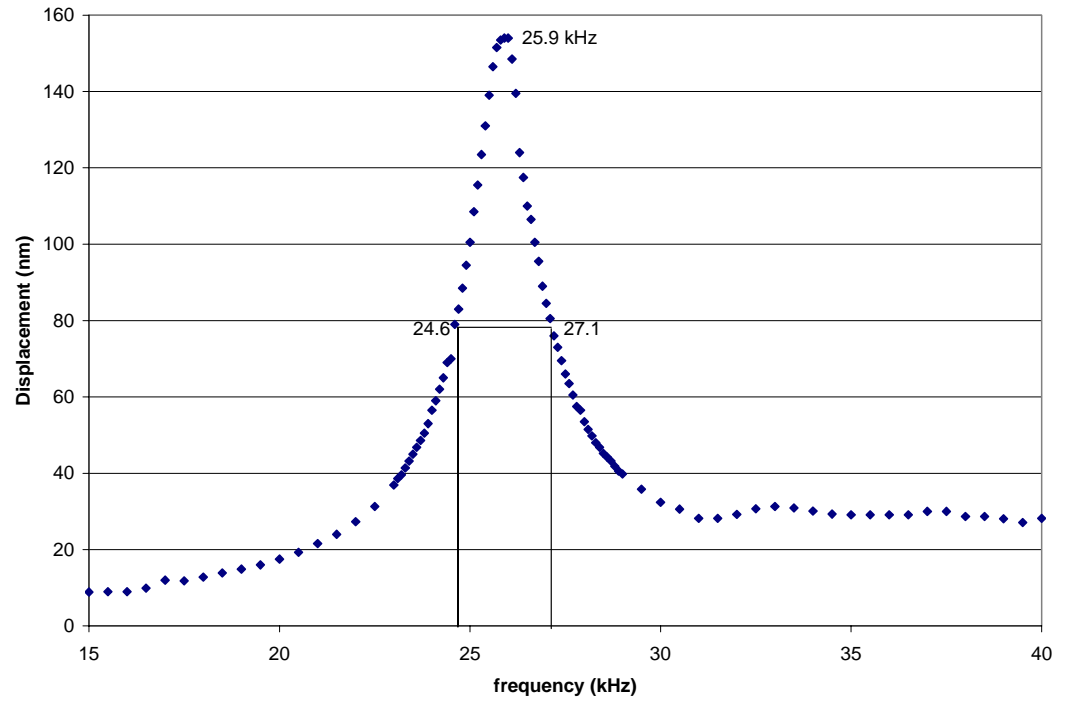

Figure 6. Frequency vs. Displacement curve for the Polycrystalline Aluminum cylinder
The resonant frequency for the Aluminum cylinder (speed of sound in Aluminum is 6400 $\mathrm{m} / \mathrm{s}$ ) is $32 \mathrm{kHz}$. It was decided to combine $599.57 \mathrm{~Hz}$ and $32 \mathrm{KHz}$ frequencies into amplitude modulated (AM) signal and use a 32 $\mathrm{kHz} \quad \mathrm{PZT}$ (leadzirconium-titanate, a piezoelectric material) transducer to excite nuclear spin resonance 
and a $1.51 \mathrm{MHz}$ transducer to excite electron spin resonance. A Wavetek signal generator was used to drive the transducers. Honey was used as the ultrasonic couplant in the experiments.

Figure 5 shows the experimental set up with the transducers attached to the Aluminum specimen. Initial experiments involved vibrating the cylinder about the resonant frequency $(32 \mathrm{kHz})$ and observing the displacements. The input voltage was 10 $\mathrm{V}$, peak-to-peak. It was observed that the peak displacement was observed at $25.9 \mathrm{kHz}$, instead of the expected $32 \mathrm{kHz}$. This is due to the fact that when the transducer is attached to the cylinder, the composite resonating length is increased and hence, there is a reduction in the resonant frequency. The displacements were observed while performing a frequency sweep about the harmonic frequency (Figure 6).

The Quality-factor (Q-factor) is given by $f / \Delta f$, where $f$ is the frequency at which the peak displacement is observed (resonant frequency) and $\Delta \mathrm{f}$ is the bandwidth at which the displacements are $50 \%$ of the peak value. The Q-factor indicates how well the sound is transmitted into the specimen. For the current set-up, it was determined to be approximately 10 . This is a very low value. To improve the Q-factor, a 3-point holder was designed and used to hold the specimen, which improved it to 16. Further efforts are underway to improve the Q-factor such as a better holder for the transducer etc. Peak displacement of $154 \mathrm{~nm}$ was observed which equates to an observed strain of 1.54 microstrains. Strains as low as 0.1 microstrains could be observed as well using the LDV.

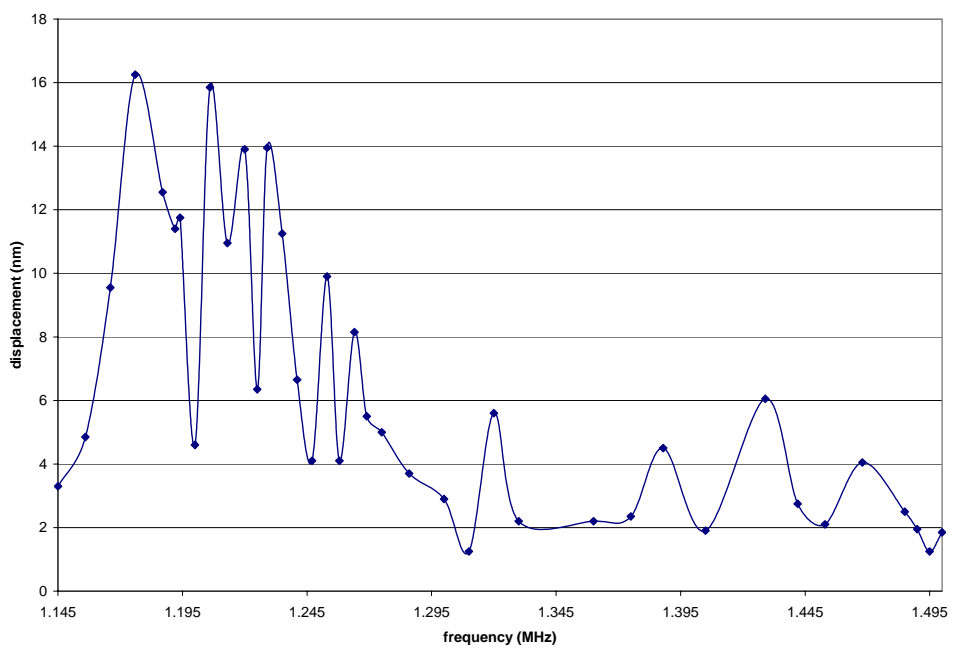

Figure 7: Frequency vs. Displacement-- $1.51 \mathrm{MHz}$ transducer attached to the cylinder 
When vibrating the cylinder with $1.511 \mathrm{MHz}$ transducer, it was very difficult to observe the displacements as $1.511 \mathrm{MHz}$ is much higher harmonic and at this frequency many different modes are also excited. This leads to not obtaining a clear frequency vs. displacement curve (Figure 7).

To identify the Q-factor of the $1.51 \mathrm{MHz}$ transducer, a thin aluminum foil was attached to the transducer and the resultant displacements were observed (Figure 8). The Q-factor was estimated to be 95 . It can be noticed that the displacements generated by the $1.51 \mathrm{MHz}$ transducer are much smaller than the $32 \mathrm{kHz}$ transducer.

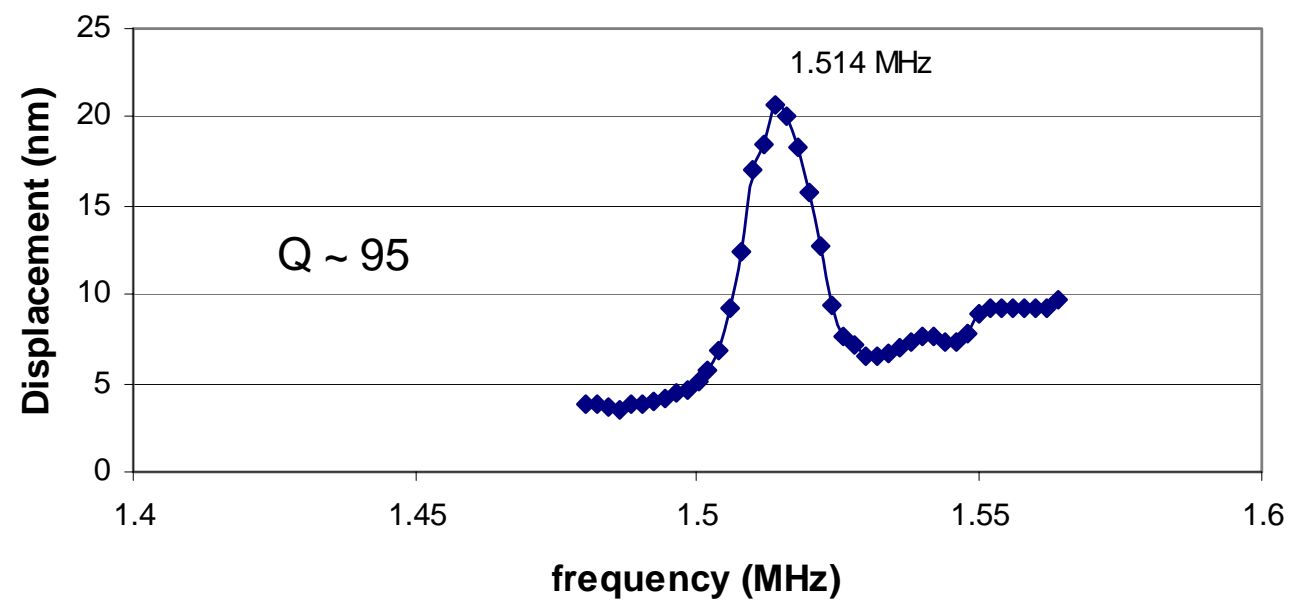

Figure 8. Q-Factor for 1.51 MHz with an aluminum foil attached to the transducer

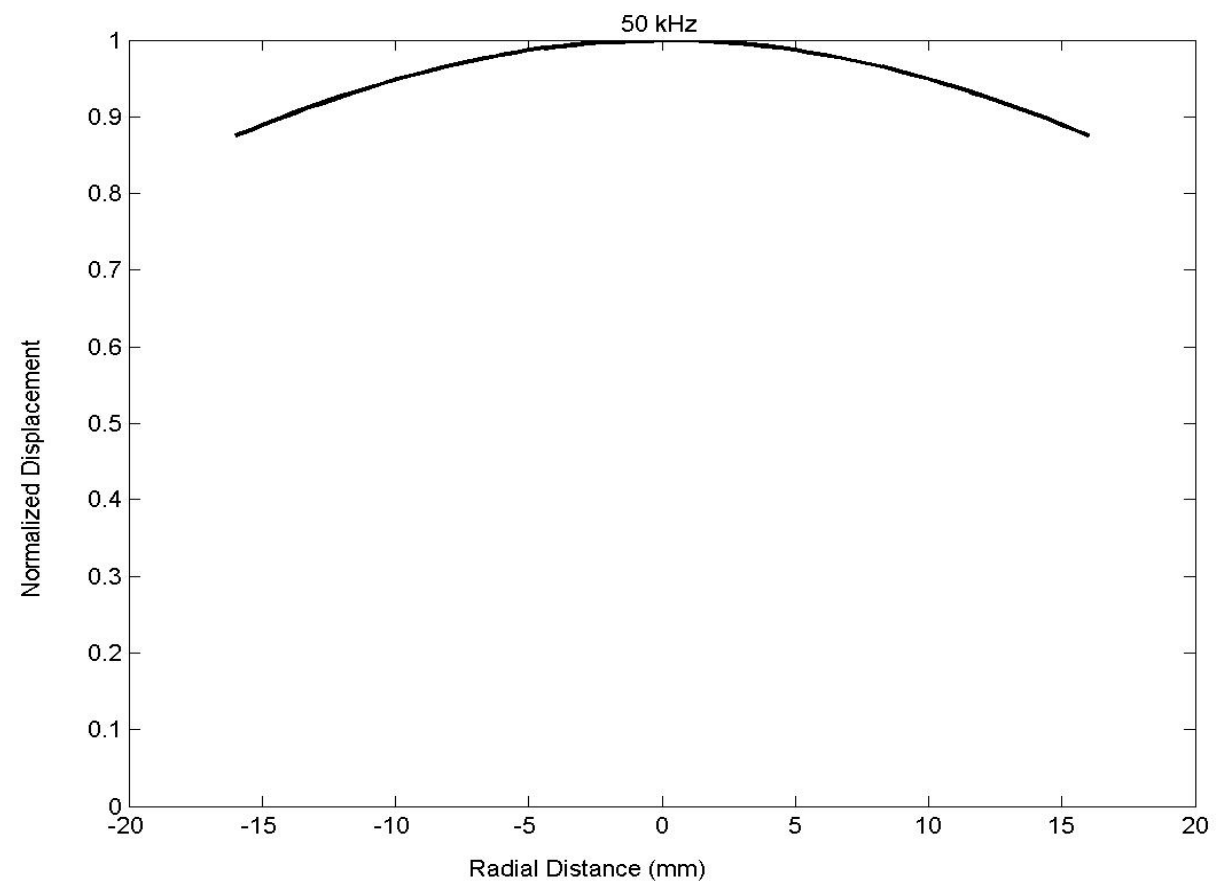

Figure 9. Simulated radial distance vs. displacement for $50 \mathrm{kHz}$ excitation 
The aluminum cylinder was vibrated using the AM signal as well and the components of the LDV signal were separated (namely in to $599 \mathrm{~Hz}$ and $32 \mathrm{kHz}$ ) and analyzed. They produced displacements similar to that shown in figure 7. An online MATLAB code, which simulates the displacements due to longitudinal vibrations in cylinders, was used to compare with the experimental data. The code helps to plot the variation in the displacements along the face of the cylinder. It was observed that, since the $32 \mathrm{kHz}$ PZT transducer was smaller than the cylinder's diameter, there was a large difference between the displacements at the outer radius and at the center of the cylinder. To validate the code, a different transducer $(50 \mathrm{kHz})$ with diameter slightly larger than the cylinder was used. These results were similar which provides confidence in the code. Those results are provided in the Figures 9-11.

Two power amplifiers have been procured which will help us in increasing the amount of strains imparted to the specimen. Efforts are underway to exactly calculate the strain energy imparted to the specimen and also understand the amount of energy required to cause the nuclear spin resonance due to the Alpher-Rubin mechanism (Dipole-dipole interaction) in aluminum.

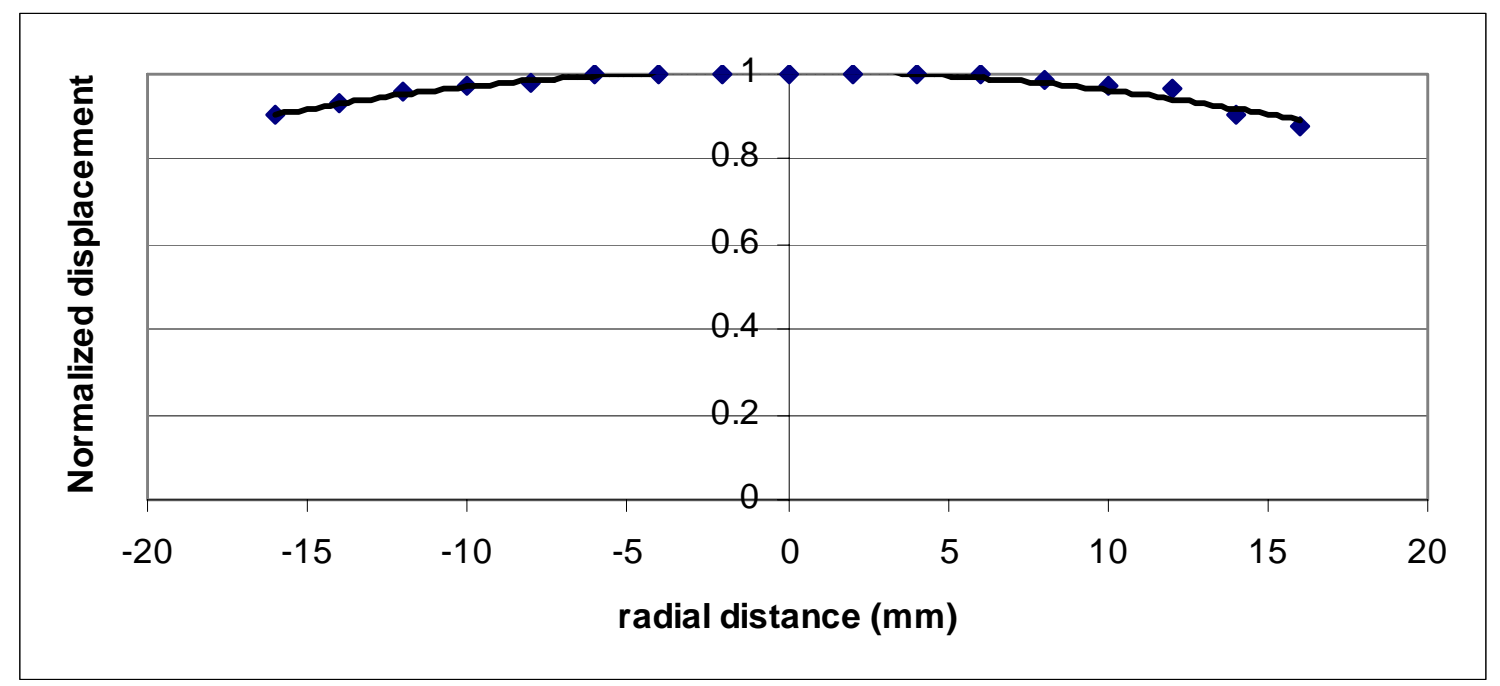

Figure 10. Experimental radial distance vs. displacement for $50 \mathrm{kHz}$ excitation

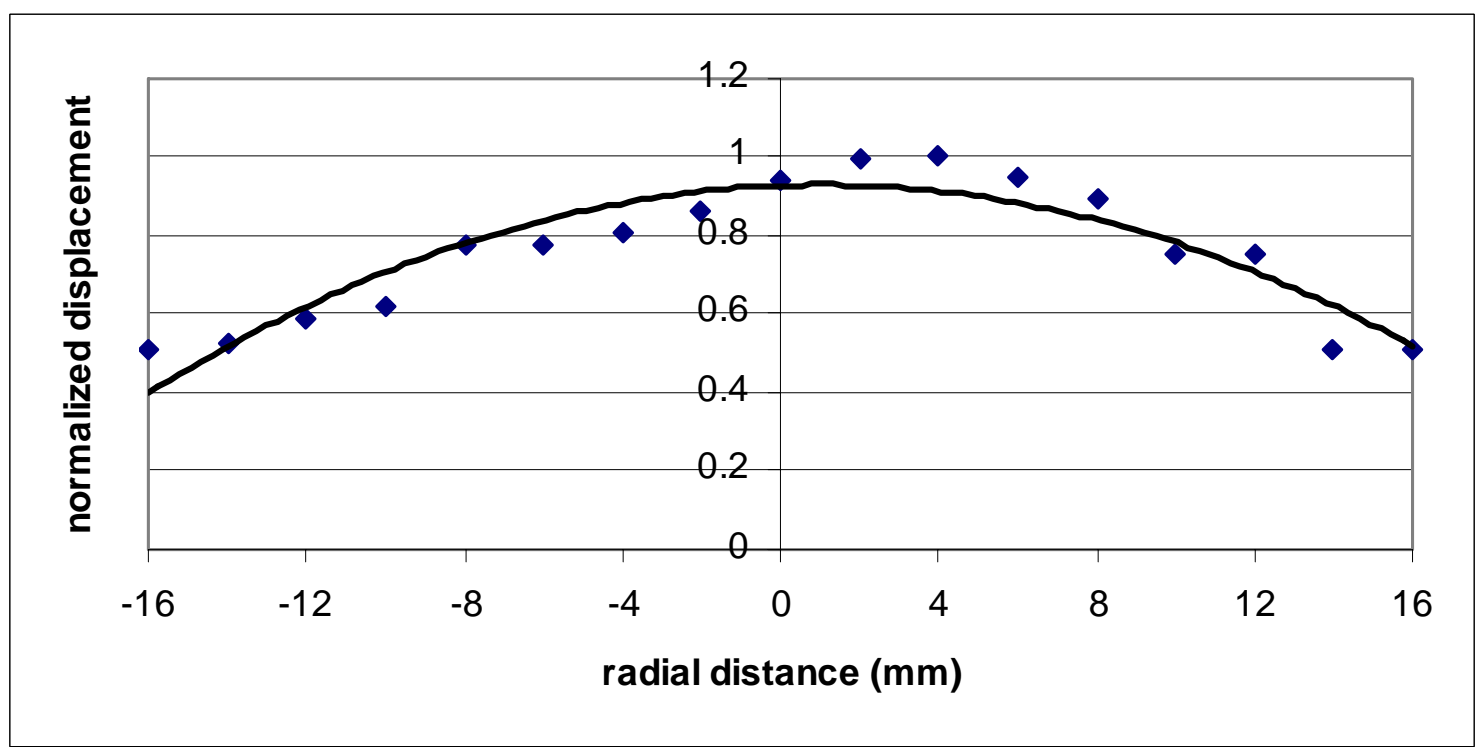

Figure 11. Experimental radial distance vs. displacement for $32 \mathrm{kHz}$ excitation 


\section{Appendix D: Begun to perform initial measurements to obtain an NAR signal for aluminum at room temperature and near the earth's magnetic field.}

Our first attempts at monitoring changes in magnetization brought about by saturating the electron and nuclear spin systems of a sample of aluminum with acoustic waves have been unsuccessful. This null result is likely due to either (1) the fact that we may only be saturating a small portion of the rather large electron spin linewidth of aluminum, or (2) the amplitude of the atomic displacement caused by the acoustic waves is not large enough to create a sufficient $b_{1}$ field.

Over the past few weeks, our efforts have been focused on demonstrating nuclear acoustic resonance (NAR) on a "simpler" sample. Dr. Ivan Hrovic, the president of GEM Systems, Inc. (the manufacturer of our potassium magnetometer), recommended that a solution of TEMPO (2,2,6,6-Tetramethylpiperidine 1-oxyl) and water would be a good system for us to start with. GEM Systems implements the Overhauser effect on a similar nitroxide at Earth's field in one of their other magnetometers. Such nitroxides offer very narrow ESR line widths and the possibility of improving on the Overhauser enhancement factor (theoretical nuclear polarization factors higher than 2000 are possible in low magnetic fields such as Earth's field).

We have made $T_{1}$ measurements on solutions with varying concentrations of TEMPO to ensure that the spin-spin relaxation time of the protons is not shortened too much (by the addition of the TEMPO) to prevent the Overhauser effect from working. At a concentration of $250 \mathrm{mg}$ of TEMPO per liter of water, $\mathrm{T}_{1}$ is only shortened to 1.35 seconds (compared to 3.6 seconds for pure de-ionized water).

Unfortunately, due to the strong hyperfine interaction of the free electron with the nitrogen nucleus, the expression for calculating the electron spin resonance (ESR) frequency of TEMPO at high fields is not valid at Earth's field. By comparing TEMPO to other similar nitroxides, we estimate the ESR frequencies to be approximately 65 MHz. In order to determine the exact frequencies needed, we have designed a "probe" consisting of a large rf coil and circuit; tunable within the range of 60-75 MHz. This method will not only determine the exact ESR frequencies, but it will prove that we will indeed be able to detect the small change in magnetization of the sample caused by saturating the spin system.

\section{Acknowledgments:}

This work was performed under the auspices of the U.S. Department of Energy by the University of California, Lawrence Livermore National Laboratory, under contract \# W7405-ENG-48. 